\title{
BRIEF
}

\section{Establishing Content Validity of a Student Pharmacist Patient Counseling Competency Assessment in Oncology}

\author{
Matthew Newman, PharmD, ${ }^{a}$ Emily Pherson, PharmD, ${ }^{a}$ Bradley Burton, PharmD, ${ }^{a}$ Eric Nemec, PharmD ${ }^{b}$ \\ ${ }^{\text {a }}$ The Johns Hopkins Hospital, Department of Pharmacy, Baltimore, Maryland \\ ${ }^{\mathrm{b}}$ Sacred Heart University, Fairfield, Connecticut
}

Corresponding Author: Matthew Newman, The Johns Hopkins Hospital, Department of Pharmacy - Carnegie 180, 600 North Wolfe St, Baltimore, MD 21287. Tel: 410-614-9806. Email: mnewma17@jhmi.edu

Submitted April 29, 2021; accepted September 13, 2021; ePublished September 2021

Objective. The main goal of this project was to establish content validity and describe internal consistency of a patient counseling competency assessment instrument used to evaluate student pharmacists practicing in an oncology setting. Methods. A modified e-Delphi panel of oncology clinical pharmacy specialists, clinical pharmacy generalists, and oncology pharmacy residents was employed. Iterative rounds of the e-Delphi process were conducted until consensus was reached on a majority of the instrument items. Consensus was defined as agreement by at least $75 \%$ of participants that an item was, or was not important. Internal consistency of defined sections of the instrument was determined using Cronbach alpha.

Results. The modified e-Delphi process included three rounds of responses from 13 panelists and resulted in a 35-item instrument with consensus reached on 33/35 (94\%) of the items, and a Cronbach alpha of 0.95. All participants indicated that the assessment result options allowed them to indicate the student's level of competency either extremely well or very well.

Conclusion. A modified e-Delphi method used to validate a reliable instrument for the assessment of student pharmacist counseling abilities in the oncology setting. Similar methodology should be considered during the development of student assessment tools, especially for high impact student pharmacist activities such as chemotherapeutic medication counseling.

Keywords: content validity, patient counseling, assessment, student pharmacists

\section{INTRODUCTION}

Student pharmacists are important resources allowing an extension of the clinical pharmacist workforce. In 2011, the Pharmacy Practice Model Initiative (PPMI) of the American Society of Health-System Pharmacists called for increased involvement of student pharmacists in drug therapy management services. ${ }^{1,2}$ Educating patients regarding the appropriate use of medications is also a core entrustable professional activity for new pharmacy graduates. ${ }^{3}$ Student pharmacists have carried out increasingly complex functions, moving from observers to integral members of the care team. Delgado and colleagues reported on student pharmacists as part of a layered learning model in an acute care setting, which included patient counseling at discharge and for targeted disease states. ${ }^{4}$ The student pharmacists allowed for expansion of pharmacy services to patients who would not otherwise have such interactions with a pharmacist. During experiential education, student pharmacists not only gain valuable experience, but also provide important clinical services to patients.

Student pharmacists completing clinical rotations (advanced pharmacy practice experiences, or APPEs) at The Johns Hopkins Hospital (JHH) are expected to provide patient counseling. Preceptors ensure each student pharmacist is sufficiently prepared and able to counsel patients as early as possible in the rotation. To promote autonomy, student pharmacists at JHH undergo patient counseling competency assessments, which cover topics relevant to their APPE (eg, internal medicine topics such as diabetes management, opioids and naloxone, and proper use of inhalers; oncology topics such as prophylactic antimicrobials and immunosuppressants for hematopoietic stem cell transplantation [HSCT]). Using a role-playing approach, the preceptor determines if the student is competent to counsel independently, requires supervision while counseling, or must repeat the assessment.

While our institution has developed instruments to evaluate student counseling competency for targeted topics, there is currently no validity evidence to support their use. In general, there is a lack of published work describing validity evidence of instruments used to assess the readiness of student pharmacists to provide patient counseling in oncology. Lin and colleagues established validity evidence for an instrument used to evaluate pharmacists providing counseling on 
herbal and dietary supplements. ${ }^{5}$ After initial creation of the instrument, pharmacists determined that the instrument demonstrated favorable reliability and construct validity. Another description of validity and reliability evidence in pharmacy education was reported by Zhou and colleagues, regarding preceptor assessment of student pharmacist performance on clinical rotations. ${ }^{6}$ While these works are important and provide a useful framework, they are not specific to the oncology setting.

The existing institution-specific counseling competency assessment instruments have been used for several years. While they are perceived as valuable among preceptors, there has not yet been a formal evaluation of the validity, content, or structure of the assessment instruments. As student pharmacists are a critical part of the pharmacy workforce and allow the pharmacy team to counsel more patients, it is important to ensure that the assessment instruments accurately reflect intended content and have the support of preceptors.

The main goal of this project was to validate the instrument to ensure the delivery of high-quality, effective, and relevant patient counseling. A secondary goal was to develop a framework to validate assessment instruments for other topics in oncology and internal medicine. The main objective was to evaluate the content validity of the assessment instrument through an e-Delphi panel of experts involved in conducting the counseling competency assessments.

\section{METHODS}

In order to evaluate the content validity of the assessment instrument, a modified e-Delphi panel process was conducted. Participants were 13 oncology clinical pharmacy specialists, generalists, and residents at JHH. This group included all known individuals within the oncology practice area of the institution who had both content expertise and experience with assessing student pharmacists for counseling activities. The project was approved by the Institutional Review Boards at Johns Hopkins Medicine.

The Delphi process is a method of systematically determining consensus among experts, consisting of several rounds of surveys or consultations. ${ }^{7}$ The use of the e-Delphi process (referring to an electronic version) has been described $^{8}$ and applied in several health care fields. ${ }^{9-12}$ Advantages of e-Delphi over traditional Delphi include the ability to maintain anonymity of panelists, the convenience of collecting responses for investigators and panelists, and the ability to include panelists in locations that may not be convenient or feasibly visited by the investigator. ${ }^{13}$ Several Delphi panels, in the traditional or modified form, have been conducted in academic and experiential pharmacy practice settings, and have focused on patient counseling, definition of competencies, and curriculum design., ${ }^{71-18}$

The topic for which the instrument was assessed is "immunosuppressants for patients undergoing HSCT." The study began with distribution of an electronic survey (Qualtrics, Provo, UT) to all panelists for e-Delphi round $1(\mathrm{~N}=13)$. Basic non-identifying descriptive data were captured, including role/job title and years of experience. In the main portion of the survey, panelists were asked to evaluate each item of the assessment instrument in use at the practice site. Panelists were asked to rate the level of importance of each item on a five-point Likert scale, and to suggest the removal of existing items or addition of new items. The estimated response time for the survey was 6-10 minutes. Panelists were asked to answer the questionnaire within 15 business days, at which point weekly email reminders were sent for an additional two weeks.

There were three of instrument evaluation conducted by the expert panel. After each round, responses were summarized, and the next round began with delivery of the summarized, anonymous responses to each panelist. Each panelist then reviewed the summarized data, compared it to their own responses, and responded to the survey in the next round. In round 1, free-text response questions were included after each survey section asking the participant if they would add any new items, or modify existing items. In round 2, panelists were presented with a summary of the free-text responses and asked if they categorically agreed or disagreed with the proposed change. If consensus was reached, the changes were made and incorporated into the round 3 survey. After rounds 1 and 2, if consensus was reached that an item was unimportant, the item was removed in the subsequent survey. The final revised instrument was shared with all panelists.

The a priori definition of consensus for this analysis was at least $75 \%$ of panelists having similar observations. For example, at least $75 \%$ of participants needed to report an item was "extremely important" or "very important" (scores of 5 and 4 on the Likert scale, respectively) for consensus to be reached that the item was important. Conversely, at least $75 \%$ of participants need to report an item was "slightly important" or "not at all important" for consensus to be reached that the item is not important. Reliability of responses in each round was described by Cronbach alpha, which has previously been applied in Delphi studies. ${ }^{19-21}$ Aggregate data were downloaded from Qualtrics and stored on a secure drive hosted by Johns Hopkins. Statistical analyses were conducted using Stata/IC 13 (StataCorp LLC, College Station, TX). 


\section{RESULTS}

The response rate for round 1 was $92 \%(\mathrm{n}=12)$. Participants included five current clinical specialists $(38 \%$ of total), three current PGY2 oncology pharmacy residents (23\%), three current pharmacist generalists (23\%), and 2 clinical specialists formerly employed by the institution (15\%). The average duration of pharmacist experience was 7 years (range 1 to 16), and average duration of oncology experience was 6 years (range 1 to 15). Most participants had previous experience administering the counseling assessment on hematopoietic cell transplantation (HCT)(58\%) or other topics $(25 \%)$.

In round 1, consensus was reached for 29 of 32 items; overall Cronbach alpha was 0.87 . The three non-consensus items were re-evaluated in round 2, after participants reviewed the summary results. A total of 21 suggestions for changes to the proposed instrument items were submitted.

In round 2, consensus was reached for 26 of 32 items, with $69 \%$ participation $(n=9)$. Of the six items that did not reach consensus, five items were removed and one revised based on qualitative feedback. Additional questions were added seeking consensus on the addition, removal, or change of items from round 1, based on the descriptive comments received. Consensus was reached for 16 of 21 proposed changes, which were incorporated into the round 3 survey. In this iteration the Cronbach alpha was 0.93 . Of the four comments submitted in round 2, none resulted in substantive changes to the survey items.

The round 3 survey had a $100 \%$ participation rate $(n=13)$ and demonstrated consensus in all but 2 items regarding the goal serum concentration ranges for tacrolimus and sirolimus, which on further review, were deemed to be important inclusions by the investigators and remained in the final assessment instrument (Table 1). The modified eDelphi process resulted in a 35-item instrument (Figure 1) with consensus reached on 33/35 (94\%) of the items, and a Cronbach alpha of 0.95 . All participants indicated that the assessment result options allowed them to indicate the student's level of competency either extremely well or very well.

\section{DISCUSSION}

Student pharmacists are capable of undertaking complex tasks including patient counseling. While standardized instruments have been used to evaluate the competency of student pharmacists to counsel patients at our institution for several years, the validity and internal consistency of the instruments had not been evaluated, nor were previously validated tools available in the literature. The authors believe applying a consistent approach to the training and assessment of student pharmacists is important to ensuring delivery of consistent, high-quality patient education. This report demonstrates the feasibility of conducting a modified e-Delphi process to establish content validity and reliability of an instrument used to assess student pharmacist counseling competency.

Content validity was established by iterative rounds of consensus decision making, as outlined by others. Internal consistency was evaluated by calculation of Cronbach alpha. The final assessment instrument yielded an alpha of 0.95 , ranging from 0.69 to 0.94 among sections of the instrument, indicating very good internal consistency.

The clinical content for this effort was immunosuppressive agents used for prophylaxis of graft rejection and graft-versus-host disease for patients undergoing allogeneic hematopoietic cell transplantation at our institution. This topic was chosen due to its overall complexity, including multiple medications, compared with other existing topics. Thus, it may not be readily generalizable to other institutions. However, it serves as a proof of concept for those using similar approaches to determining competency of student pharmacists providing patient counseling. While most participants had experience using the instrument, several years elapsed between original implementation and this work, including staff turnover, which we believe allowed for objective participation. It is expected that previous experience assessing students with the instrument also provided valuable context and subject matter expertise in which participants considered necessary changes.

The modified e-Delphi method was straightforward in its design and application, using survey software and email communication. The modified e-Delphi process may be prohibitively labor-intensive to apply to all of the various topics on which student pharmacists may be assessed. It may be most useful to use this approach to establish validity of complex topics, or those for which there is subjective controversy; then, employ the institution specific framework to revise the assessment of other, more straightforward topics. The high participation rate for our surveys indicates that stakeholders had a clear interest in ensuring the appropriateness of the instrument. However, it may become burdensome to repeat the process several times. Students are currently assessed on topics chosen at the discretion of their preceptor, based on the needs of patients during the rotation. This creates an opportunity for specific groups of preceptors to focus on topics that may be most relevant to their practice area.

The revised instrument is now used for assessment of student pharmacists. Future directions for this research include applying the modified e-Delphi process to other assessment instruments for student pharmacists at our institution. By establishing validity of one specialized instrument, additional instruments may undergo a similar process in 
order for pharmacy preceptors be confident that student pharmacists are evaluated on appropriate criteria. With proven consensus and high internal consistency demonstrated, future studies may determine the effectiveness of patient counseling conducted by student pharmacists, and evaluation of performance with patients compared with initial roleplaying assessment with the preceptor.

\section{CONCLUSION}

A modified e-Delphi method was employed to establish content validity and internal consistency of a frequently used assessment instrument for student pharmacists participating in patient counseling in oncology. While the end result is an improved assessment instrument for counseling patients on immunosuppression for HCT, this work also serves as a proof of concept and feasibility study. The modified e-Delphi method may be used to establish content validity for assessment instruments in other areas of patient counseling, including content areas that may be complex or controversial.

\section{ACKNOWLEDGMENTS}

This work is the result of a capstone project for the Master of Education in Health Professions program at The Johns Hopkins University School of Education. The authors are grateful for the participation of the panelists and their ongoing commitment to training student pharmacists.

\section{REFERENCES}

1. The consensus of the Pharmacy Practice Model Summit. Am J Health Syst Pharm. 2011;68(12):1148-1152. doi:10.2146/ajhp110060

2. Executive summary. Am J Health Syst Pharm. 2011;68(12):1079-1085. doi:10.2146/ajhp110110

3. Haines ST, Pittenger AL, Stolte SK, et al. Core Entrustable Professional Activities for New Pharmacy Graduates. Am J Pharm Educ. 2017;81(1). doi:10.5688/ajpe811S2

4. Delgado O, Kernan WP, Knoer SJ. Advancing the pharmacy practice model in a community teaching hospital by expanding student rotations. Am J Health Syst Pharm. 2014;71(21):1871-1876. doi:10.2146/ajhp130624

5. Lin H-W, Pickard AS, Mahady GB, Karabatsos G, Crawford SY, Popovich NG. An Instrument to Evaluate Pharmacists' Patient Counseling on Herbal and Dietary Supplements. Am J Pharm Educ. 2010;74(10). Accessed December 17, 2019. https://www.ncbi.nlm.nih.gov/pmc/articles/PMC3058472/

6. Zhou L, Almutairi AR, Alsaid NS, Warholak TL, Cooley J. Establishing the Validity and Reliability Evidence of Preceptor Assessment of Student Tool. Am J Pharm Educ. 2017;81(8). doi:10.5688/ajpe5908

7. Benson H, Lucas C, Williams KA. Establishing consensus for general practice pharmacist education: A Delphi study. Curr Pharm Teach Learn. 2020;12(1):8-13. doi:10.1016/j.cpt1.2019.10.010

8. The Delphi Technique in Nursing and Health Research. 1st ed. John Wiley \& Sons, Ltd; 2011. doi:10.1002/9781444392029

9. Msibi PN, Mogale R, de Waal M, Ngcobo N. Using e-Delphi to formulate and appraise the guidelines for women's health concerns at a coal mine: A case study. Curationis. 2018;41(1). doi:10.4102/curationis.v41i1.1934

10. Guerreiro MP, Plácido M, Barros CT, et al. A national e-Delphi towards the measurement of safe medication practices in Portuguese hospitals. Eur J Hosp Pharm. 2018;25(2):103-106. doi:10.1136/ejhpharm-2016-000955

11. Taylor RM, Feltbower RG, Aslam N, Raine R, Whelan JS, Gibson F. Modified international e-Delphi survey to define healthcare professional competencies for working with teenagers and young adults with cancer. BMJ Open. 2016;6(5):e011361. doi:10.1136/bmjopen-2016-011361

12. Avery A, Savelyich B, Sheikh A, et al. Identifying and establishing consensus on the most important safety features of GP computer systems: e-Delphi study. J Innov Health Inform. 2005;13(1):3-11. doi:10.14236/jhi.v13i1.575

13. Donohoe H, Stellefson M, Tennant B. Advantages and Limitations of the e-Delphi Technique. Am J Health Educ. 2012;43(1):38-46. doi:10.1080/19325037.2012.10599216

14. Ignoffo R, Chan L, Knapp K, et al. Efficient and effective precepting of pharmacy students in acute and ambulatory care rotations: A Delphi expert panel study. Am J Health Syst Pharm. 2017;74(19):1570-1578. doi:10.2146/ajhp170181

15. Puumalainen II, Kause JM, Airaksinen MS. Quality Assurance Instrument Focusing on Patient Counseling. Ann Pharmacother. 2005;39(7-8):1220-1226. doi:10.1345/aph.1E629

16. Covvey JR, Ryan M. Use of a Modified Delphi Process to Determine Course Objectives for a Model Global Health Course in a Pharmacy Curriculum. Am J Pharm Educ. 2018;82(8). doi:10.5688/ajpe6358

17. Traynor AP, Borgelt L, Rodriguez TE, Ross LA, Schwinghammer TL. Use of a Modified Delphi Process to Define the Leadership Characteristics Expected of Pharmacy Faculty Members. Am J Pharm Educ. 2019;83(7). doi:10.5688/ajpe7060 
18. Janke KK, Kelley KA, Sweet BV, Kuba SE. A Modified Delphi Process to Define Competencies for Assessment Leads Supporting a Doctor of Pharmacy Program. Am J Pharm Educ. 2016;80(10). doi:10.5688/ajpe8010167

19. Graham B, Regehr G, Wright JG. Delphi as a method to establish consensus for diagnostic criteria. J Clin Epidemiol Elmsford. 2003;56(12):1150-1156. doi:http://dx.doi.org.proxy1.library.jhu.edu/10.1016/S0895-4356(03)00211-7

20. Tomasik T. Reliability and validity of the Delphi method in guideline development for family physicians. Qual Prim Care. 2010;18(5):317-326.

21. Collins JW, Levy J, Stefanidis D, et al. Utilising the Delphi Process to Develop a Proficiency-based Progression Train-the-trainer Course for Robotic Surgery Training. Eur Urol. 2019;75(5):775-785. doi:10.1016/j.eururo.2018.12.044 
Table 1. Summary of e-Delphi Round 3 Responses

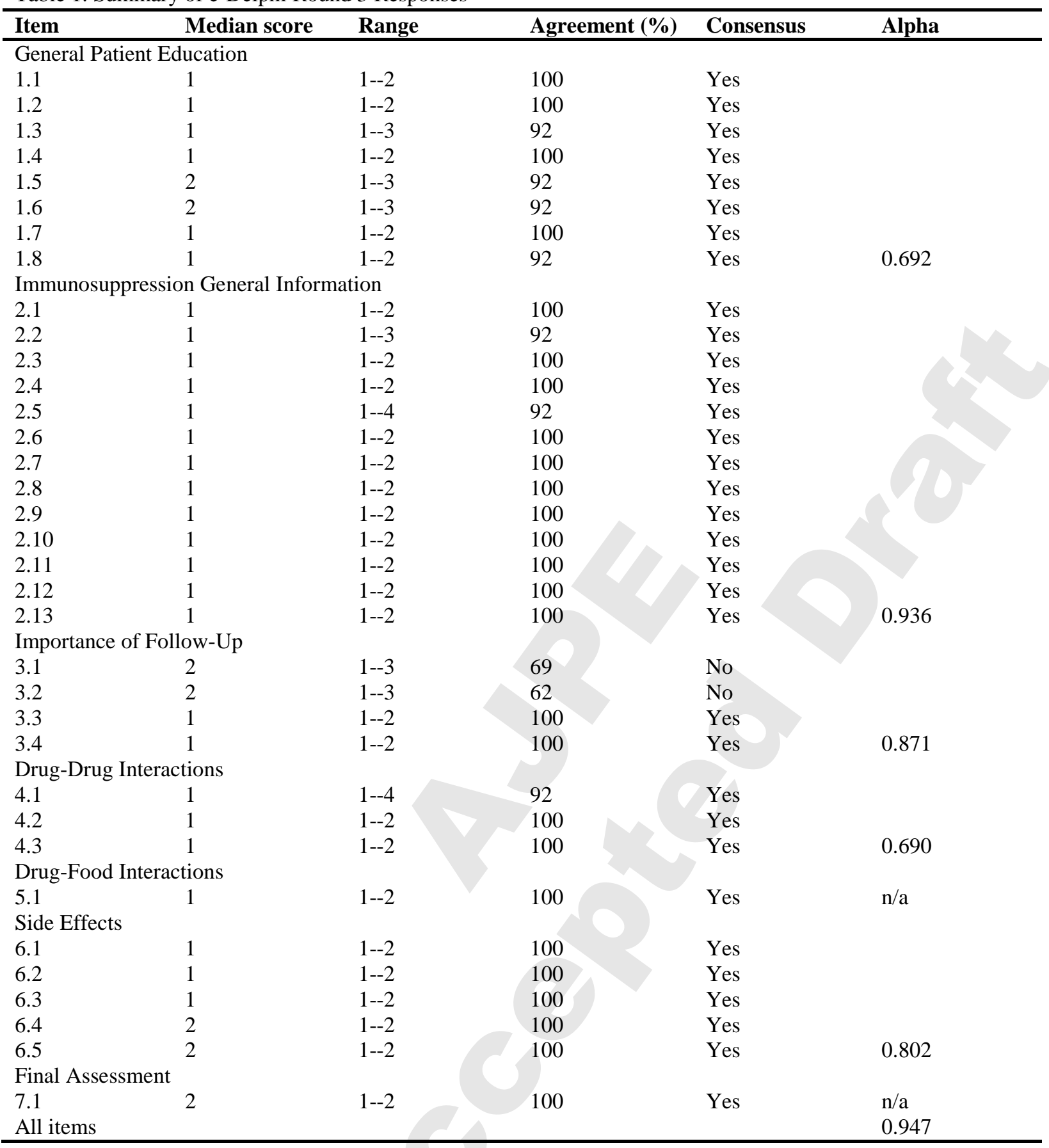


Figure 1. Learner Competency for Hematopoietic Stem Cell Transplant Immunosuppression

\begin{tabular}{|c|c|}
\hline \begin{tabular}{|l|l|} 
Category \\
\end{tabular} & Essential Components \\
\hline $\begin{array}{l}\text { General Patient } \\
\text { Education }\end{array}$ & $\begin{array}{l}\square \text { Correctly identifies the patient } \\
\square \quad \text { Introduces self } \\
\square \quad \text { Explains purpose of education } \\
\square \text { Uses plain language and medical terms the patient can understand } \\
\square \quad \text { Uses open-ended questions } \\
\square \quad \text { Asks patient to explain in their own terms what was understood using teach-back questions } \\
\square \quad \text { Identifies gaps in understanding and provides clarification } \\
\square \quad \text { Answers questions within their scope, defers to physician or pharmacist when appropriate }\end{array}$ \\
\hline 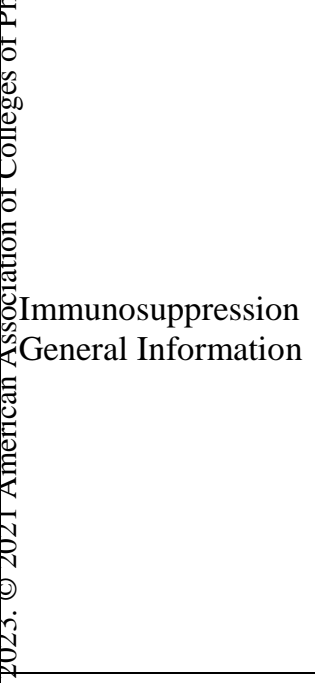 & $\begin{array}{l}\square \quad \text { Choose the correct counseling handout } \\
\square \quad \text { Reviews indication (prevention of GVHD and graft rejection) } \\
\square \quad \text { Reviews dosing and administration for MMF } \\
\square \quad \text { Three times daily } \\
\square \quad 1 \text { hour before or } 2 \text { hours after or any supplements that contains calcium, aluminum, } \\
\text { magnesium, or iron including antacids } \\
\square \quad \text { Taken from Day }+5 \text { through Day }+35 \text { ( } 31 \text { days total) } \\
\square \quad \text { Reviews dosing and administration for tacrolimus } \\
\square \quad \text { If PO, q } 12 \text { hours, consistently with or without food; if IV, daily } \\
\square \quad \text { Starts Day }+5 \text { and continues for } 2 \text { to } 6 \text { months after transplant. Do not stop until } \\
\quad \text { instructed. } \\
\square \quad \text { Brand and generic tacrolimus are not be interchanged } \\
\square \quad \text { Reviews dosing and administration for sirolimus } \\
\square \quad \text { Taken daily } \\
\square \quad \text { Starts Day }+5 \text { and continues for } 2 \text { to } 6 \text { months after transplant. Do not stop until } \\
\text { instructed. }\end{array}$ \\
\hline $\begin{array}{l}\text { EImportance of } \\
\text { Fillow-up } \\
\text { Foll }\end{array}$ & $\begin{array}{ll}\square & \text { Tacrolimus goal (typically } 10-15 \mathrm{ng} / \mathrm{mL} \text { ); provider may change goal } \\
\square & \text { Sirolimus goal (typically } 5-15 \mathrm{ng} / \mathrm{mL} \text { ); provider may change goal } \\
\square & \text { Explains reasons behind importance of keeping appointments for lab draws } \\
\square & \text { Reminds patient not to take morning dose when in clinic on the day levels are to be drawn }\end{array}$ \\
\hline 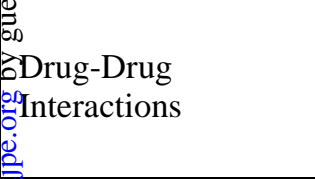 & $\begin{array}{l}\square \text { MMF: supplements that contain calcium, aluminum, magnesium, or iron } \\
\square \text { Tacrolimus: certain antifungals, blood pressure medications, and herbal medications } \\
\square \text { Tells patient to ask provider before starting new medications or stopping any medications as } \\
\text { there may be impact on immunosuppressant drug levels }\end{array}$ \\
\hline $\begin{array}{l}\text { Drug-Food } \\
\text { Interactions }\end{array}$ & $\square$ Tacrolimus: avoid grapefruit, Seville orange, star fruit \\
\hline Side Effects & $\begin{array}{ll} & \text { MMF: nausea, vomiting or diarrhea } \\
\square & \text { Tacrolimus: Mild tremors/shakiness, high heart rate or blood pressure, high blood sugar, } \\
\text { changes in vision, kidney problems; may require additional medications to manage } \\
\square \quad \text { Sirolimus: swelling, high cholesterol or triglycerides, sore muscles or joints, mouth sores } \\
\square \quad \text { Providers will be monitoring for side effects } \\
\square \quad \text { List presented here not all-inclusive; report any new symptoms to provider } \\
\end{array}$ \\
\hline Final Assessment & $\begin{array}{l}\square \text { Student is competent to counsel without observation } \\
\square \quad \text { Student should be observed prior to counseling on his/her own } \\
\square \text { Student needs additional practice with a preceptor prior to counseling on his/her own }\end{array}$ \\
\hline
\end{tabular}

